

\title{
Optimisation du réseau de transfert thermique des centrales solaires à convertisseurs héliothermiques distribués. - Application aux capteurs de type THEK R. Pasquetti
}

\section{- To cite this version:}

R. Pasquetti. Optimisation du réseau de transfert thermique des centrales solaires à convertisseurs héliothermiques distribués. - Application aux capteurs de type THEK. Revue de Physique Appliquée, 1979, 14 (1), pp.35-47. 10.1051/rphysap:0197900140103500 . jpa-00244589

HAL Id: jpa-00244589

https://hal.science/jpa-00244589

Submitted on 1 Jan 1979

HAL is a multi-disciplinary open access archive for the deposit and dissemination of scientific research documents, whether they are published or not. The documents may come from teaching and research institutions in France or abroad, or from public or private research centers.
L'archive ouverte pluridisciplinaire HAL, est destinée au dépôt et à la diffusion de documents scientifiques de niveau recherche, publiés ou non, émanant des établissements d'enseignement et de recherche français ou étrangers, des laboratoires publics ou privés. 


\title{
Optimisation du réseau de transfert thermique des centrales solaires à convertisseurs héliothermiques distribués.
}

\section{Application aux capteurs de type THEK}

\author{
R. Pasquetti \\ Département d'Héliophysique $\left(^{*}\right)$, Université de Provence, \\ Centre de Saint-Jérôme, 13397 Marseille Cedex 4, France
}

(Reçu le 23 octobre 1978, accepté le 27 octobre 1978)

\begin{abstract}
Résumé. - Cet exposé concerne les centrales à convertisseurs distribués de l'énergie solaire en chaleur ; ainsi, le réseau de transfert thermique, capteurs-unité de production énergétique, fait, une fois défini, l'objet d'une optimisation. La démarche de calcul proposée est, à cet effet, appliquée à des centrales qui seraient composées d'héliostats de type THEK (4 à 100 000) implantés sur un site météorologiquement comparable à celui de Carpentras. La prédétermination du positionnement des capteurs résulte, tout d'abord, de l'étude des effets d'ombre portée. Les différentes pertes énergétiques et le coût du réseau de connexion sont ensuite formulées en fonction des dimensions caractéristiques du système. En dernier lieu, un bilan physico-économique conduit à des graphes permettant, pour tout type d'exploitation, l'optimisation du réseau de transfert thermique des centrales étudiées. La méthode mise au point est utilisée pour des unités de divers dimensionnements et trois exploitations de nature différente : production de chaleur, d'électricité, d'énergie totale. On précise, de plus, les performances de ces centrales, et l'on évalue leur dimensionnement optimal et maximal.
\end{abstract}

\begin{abstract}
This study is concerned with power plants based on a distributed network of collectors to convert solar energy into heat ; the fluid transport network, between collectors and the central production unit, is defined and then optimized. The method of calculation is applied to power plants using from 4 to 100000 collectors of the THEK type, installed on a site with meteorological conditions similar to those of the Carpentras' area. The predetermination of the collectors position is made by the study of shadow effects. The variations of the different energy losses and of the network cost, with the caracteristic dimensions of the system, are then expressed. Taking into account physical and economical parameters, graphs are given wich can be used for the optimization of the fluid transport network of THEK power plants. Finally, these graphs are used for different sizes of power plants in three cases of energy production : heat, electricity, and " total energy " production. The optimal and maximal size of these types of power plants, as well as their yields, are defined in each of the three cases.
\end{abstract}

1. Introduction. - Les centrales solaires à convertisseurs distribués étudiées ici utilisent la voie thermodynamique pour la conversion de l'énergie solaire. Ces centrales comprennent, par définition, un ensemble de capteurs au niveau de chacun desquels s'effectue la conversion héliothermique. C'est un fluide caloporteur approprié qui permet, grâce à un réseau de transfert adéquat, le transfert de l'énergie reçue à une installation productrice de chaleur ou d'électricité. Précédemment, l'énergie captée peut transiter par un réservoir de stockage. Un tel ensemble est schématisé figure 1 .

Nous proposons dans cet exposé une méthode d'optimisation du réseau de transfert capteurs-conver-

(*) Equipe de Recherche associée au C.N.R.S. $\mathrm{n}^{\circ} 538$. tisseur thermique ou thermo-électrique ; conséquemment, on évaluera le dimensionnement optimal et

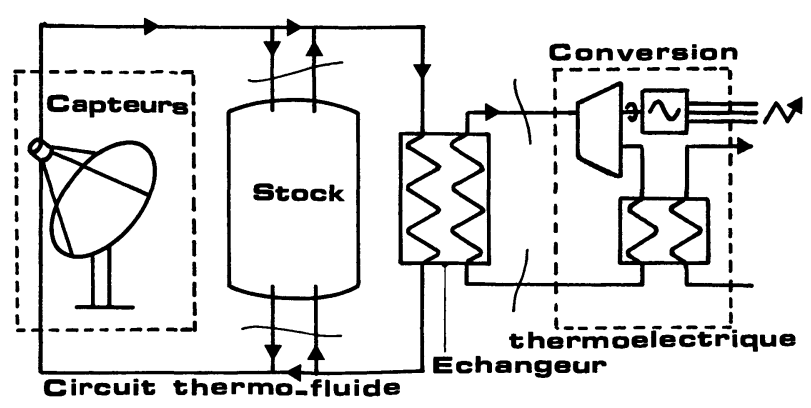

Fig. 1. - Schéma de principe d'un ensemble de conversion par voie thermodynamique de l'énergie solaire.

[Schematic of a thermodynamical system to convert solar energy.] 
maximal de ce type de centrale. La méthode sera appliquée aux capteurs développés dans le cadre du projet THEK [1].

Le capteur de type THEK, destiné à fonctionner à des niveaux de température élevés, supérieurs à $200{ }^{\circ} \mathrm{C}$, concentre l'énergie incidente avant de la convertir. Cet héliostat possède une surface réfléchissante pseudo-paraboloïdale, pointée en permanence vers le soleil, et ses caractéristiques sont les suivantes :

- surface de captage : $50 \mathrm{~m}^{2}$,

- concentration géométrique : 230 ,

- fluide caloporteur monophasique pour le cycle thermique considéré,

- température approximative d'entrée et de sortie au convertisseur : 200 et $300{ }^{\circ} \mathrm{C}$,

- rendement de conversion héliothermique voisin de 0,7 .

Les capteurs seront de plus supposés être circulaires et implantés dans un terrain plan d'ensoleillement connu. La démarche proposée est toutefois d'une plus grande généralité et reste applicable quels que soient les paramètres caractéristiques $\mathrm{du}$ capteur, de son utilisation, ou de son implantation.

Les principaux éléments à retenir dans l'étude du réseau de transfert thermique d'une centrale à convertisseurs distribués sont :

- les effets d'ombre portée entre capteurs,

- la puissance de pompage nécessaire à la circulation du fluide,

- les pertes thermiques en ligne le long des canalisations constituant le réseau de transfert thermique,

- l'inertie thermique de ce réseau de transfert (due aux périodes de non-fonctionnement),

\section{- son coût.}

Les quatre derniers éléments tendent à induire une proximité aussi grande que possible entre héliostats, tandis que les effets d'ombre portée entrâ̂nent un effet contraire. La possibilité d'une optimisation du réseau de transfert thermique est donc bien évidente. De plus, le premier élément n'est fonction que de la position relative des capteurs et non pas de la manière dont ils sont connectés ; une prédétermination du positionnement des héliostats par l'étude des effets d'ombre pourra ainsi être envisagée en premier lieu, dans le paragraphe 1.

Le choix d'un mode de connexion des capteurs et l'étude du réseau de transfert thermique ainsi défini feront l'objet du second paragraphe.

Enfin, l'optimisation sur critères physico-économiques du système sera effectuée dans le paragraphe 3 .

1. Prédétermination du positionnement des héliostats : étude des effets d'ombre. - L'implantation des capteurs devra évidemment présenter une symétrie par rapport à un axe Nord/Sud. De plus, si l'on désire une répartition régulière sur le terrain, les héliostats ne peuvent être positionnés que de deux manières différentes : en rectangle ou en losange.

Dans chacune des possibilités évoquées, l'implantation peut alors être caractérisée par la valeur de deux coefficients adimensionnés (Fig. 2) :

- le coefficient d'espacement $(\tau)$ directement proportionnel au taux d'occupation au sol, c'est-à-dire au rapport de la surface de miroirs à la surface de l'installation,

- le coefficient de déformation $\left(\tau^{\prime}\right)$, caractéristique de la déformation de la maille, sans entraîner une variation de sa surface.

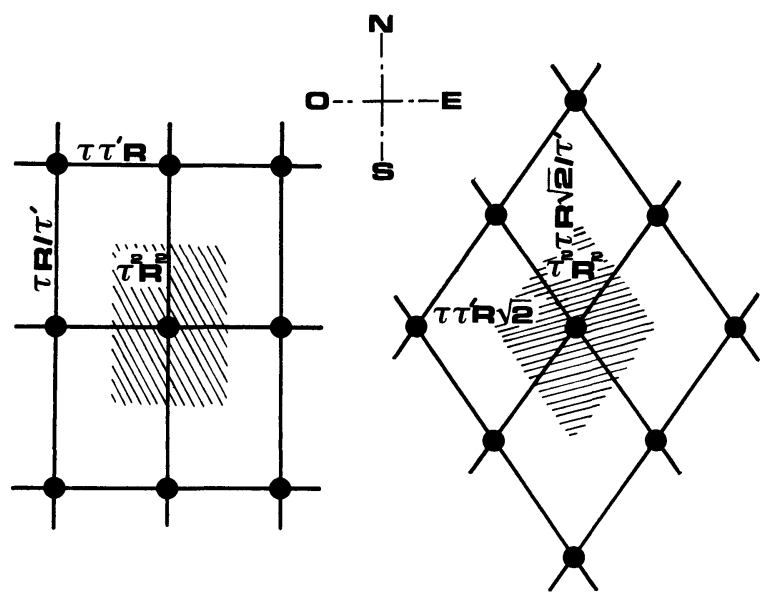

Fig. 2. - Configurations en rectangle et en losange ; définition des coefficients d'espacement et de déformation $\tau$ et $\tau^{\prime}$. $R$ est le rayon du disque de captage et $\tau^{2} R^{2}$ la surface de terrain par capteur.

[Rectangle and lozenge disposition; definition of the spacing and deformation coefficients $\tau$ and $\tau^{\prime}$. $R$ is the receiving disc radius and $\tau^{2} R^{2}$ the land surface for one collector.]

Remarquons que le taux d'occupation au sol $\left(\tau_{\mathrm{s}}\right)$ est tel que : $\tau_{\mathrm{s}}=\pi / \tau^{2}$.

Supposons connu, à un instant et en un lieu donnés, le flux solaire incident direct $(H)$; on peut en déduire le flux solaire effectivement reçu $\left(H_{\mathrm{e}}\right)$ par un capteur. Pour une latitude déterminée, on a :

$$
\begin{aligned}
& H_{\mathrm{e}}\left(a_{\mathrm{h}}, \delta, \tau, \tau^{\prime}\right)=\frac{S-\Delta S\left(a_{\mathrm{h}}, \delta, \tau, \tau^{\prime}\right)}{S} \times \\
& \quad \times H\left(a_{\mathrm{h}}, \delta\right) \gamma\left(a_{\mathrm{h}}\right)
\end{aligned}
$$

avec $\gamma$ non nul et égal à 1 seulement si le capteur est en fonctionnement; où $S$ est la surface de captage, $\Delta S$ la partie ombrée de $S, \delta$ la déclinaison et $a_{\mathrm{h}}$ l'angle horaire, proportionnel au temps $(t)$.

Les intégrales des fonctions $\gamma H$ et $H_{\mathrm{e}}$, par rapport à $t$, conduisent aux valeurs de l'énergie incidente récupérable $(\bar{H})$ et de l'énergie incidente effectivement captée $\left(\bar{H}_{\mathrm{e}}\right)$ par unité de surface de capteur et pour une journée particulière.

On peut alors définir un coefficient journalier de captage $\left(\xi_{\delta}\right)$ tel que : 
$\xi_{\delta}\left(\delta, \tau, \tau^{\prime}\right)=\frac{\bar{H}_{\mathrm{e}}}{\bar{H}}$ avec $\bar{H}_{\mathrm{e}}=\frac{\mathrm{d} t}{\mathrm{~d} a_{\mathrm{h}}} \int_{0}^{2 \pi} H_{\mathrm{e}} \mathrm{d} a_{\mathrm{h}}$

et

$\bar{H}=\frac{\mathrm{d} t}{\mathrm{~d} a_{\mathrm{h}}} \int_{0}^{2 \pi} \gamma H \mathrm{~d} a_{\mathrm{h}}$.

D'une manière similaire, il est possible de définir un coefficient annuel de captage $(\xi)$ :

$\xi\left(\tau, \tau^{\prime}\right)=\frac{\overline{\bar{H}_{\mathrm{e}}}}{\overline{\bar{H}}}$ avec $\overline{\bar{H}}_{\mathrm{e}}=\sum_{\text {année }} \bar{H}_{\mathrm{e}}$

et

$\overline{\bar{H}}=\sum_{\text {année }} \bar{H}$.

Dans la suite de cet exposé, les effets de bord de l'implantation seront négligés : les coefficients $\xi_{\delta}$ et $\xi$ seront supposés indépendants du capteur envisagé. Nous obtiendrons ainsi, notamment pour les centrales les plus petites, un résultat par défaut.

Théoriquement, c'est l'étude du coefficient annuel de captage, fonction des seuls paramètres caractéristiques du champ et de l'année envisagée, qui permet une prédétermination satisfaisante.

Pratiquement, il est toutefois incommode de suivre la démarche proposée ci-dessus ; les relevés météorologiques ne sont en effet généralement pas suffisamment fins et il est par ailleurs souhaitable que $\xi$ ne soit fonction que des paramètres $\tau$ et $\tau^{\prime}$.

Pour cette raison, nous avons adopté pour $H\left(a_{\mathrm{h}}, \delta\right)$ des valeurs théoriques, supposant des journées parfaitement ensoleillées et un coefficient de trouble du ciel égal à 0,1 . Si $\varphi$ est la latitude du lieu, on a [2] :

$$
\begin{aligned}
& H\left(a_{\mathrm{h}}, \delta\right)=1160 \exp (-1 / 4,5(\sin \varphi \sin \delta- \\
& \left.\left.-\cos \varphi \cos \delta \cos a_{\mathrm{h}}\right)\right)
\end{aligned}
$$

De la même manière que pour $\bar{H}$ et $\bar{H}_{\mathrm{e}}$, le coefficient journalier de captage $\left(\xi_{\delta}\right)$, calcule d'après (2), correspond à une journée type et n'est plus fonction de l'année envisagée.

La détermination de $\xi$ nécessite pourtant de tenir compte des différentes probabilités d'ensoleillement selon les périodes de l'année. On peut alors substituer à (3) les formulations suivantes de $\overline{\bar{H}}_{\mathrm{e}}$ et $\overline{\bar{H}}$ :

$\overline{\bar{H}}_{\mathrm{e}}=\sum_{\text {année }} q(\delta) \bar{H}_{\mathrm{e}}$

et

$\overline{\bar{H}}=\sum_{\text {année }} q(\delta) \bar{H}$

où $q$ est, pour une période de l'année, la probabilité d'ensoleillement, et $\delta$ la valeur moyenne de la déclinaison au cours de cette période. Les valeurs de $q$ étant déterminées d'après des relevés effectués sur un nombre important d'années, le coefficient annuel de captage ne dépend plus que des coefficients $\tau$ et $\tau^{\prime}$.
Sur la figure 3 sont représentées, pour la configuration en losange et avec des données particulières, les variations de $H_{\mathrm{e}}$ en fonction du temps ; les effets d'ombre dus aux capteurs non voisins de celui étudié, ainsi que l'incidence du rayonnement de seuil des héliostats ont ici été négligés.

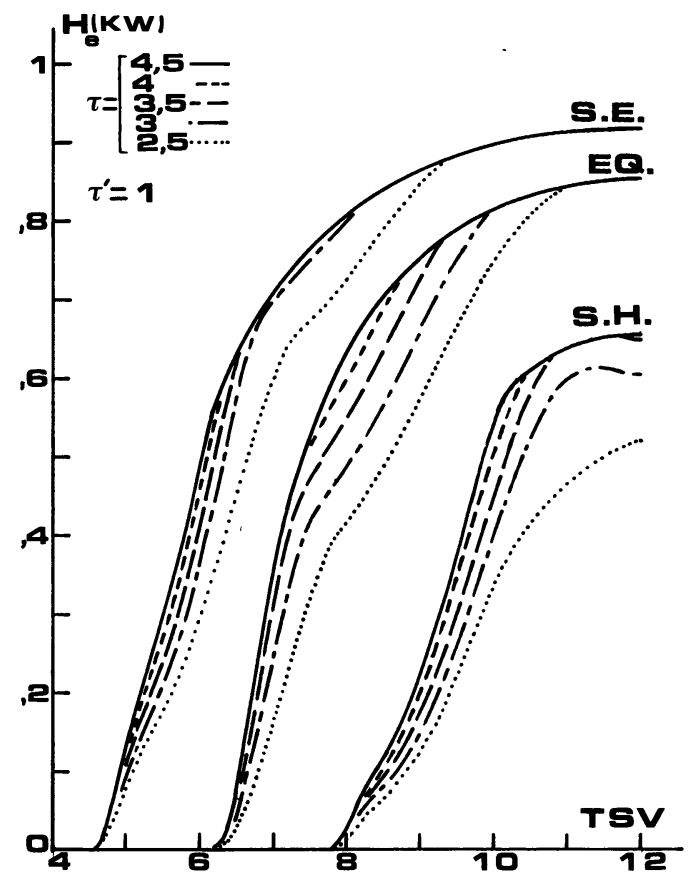

Fig. 3. - Variations, avec le temps solaire vrai, de la puissance solaire incidente captée pour la configuration en losange, un coefficient de déformation égal à 1 et diverses valeurs du coefficient d'espacement; les valeurs de la déclinaison correspondent à l'équinoxe et aux solstices; la latitude est égale à $43^{\circ} 20^{\prime}$.

[Variations of the collected solar power, versus solar time, for the lozenge disposition, a deformation coefficient equal to 1 and various values of the spacing coefficient; the declination corresponds to equinox and solstices; the latitude is equal to $43^{\circ} 20^{\prime}$.]

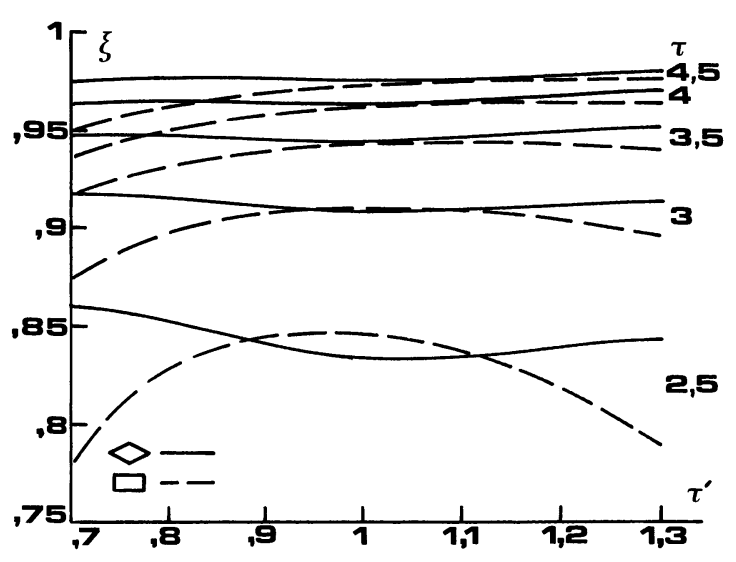

Fig. 4. - Variations du coefficient annuel de captage avec le coefficient de déformation pour les configurations en rectangle et en losange et pour diverses valeurs du coefficient d'espacement.

[Variations of the annual collecting coefficient, versus the deformation coefficient, for the rectangle and lozenge dispositions and various values of the spacing coefficient.] 
Figure 4 apparaissent les variations de $\xi$ en fonction de $\tau^{\prime}$, pour les configurations en losange et en rectangle, et pour diverses valeurs de $\tau$. Les probabilités d'ensoleillement ont été prises égales au rapport des moyennes mensuelles de la durée réelle à la durée maximale d'insolation à Carpentras [3]. Ces valeurs sont données dans le tableau I.

Tableau I

\begin{tabular}{|c|c|c|c|c|c|c|}
\hline \multirow{4}{*}{$q(\%)$} & Janvier & Février & Mars & Avril & Mai & Juin \\
\hline & $4 \overline{49,45}$ & $\overline{59,80}$ & 55,56 & $5 \overline{59,85}$ & $\overline{60,00}$ & 67,76 \\
\hline & 55,68 & 50,00 & 65,14 & 62,40 & 76,09 & 79,33 \\
\hline & Déc. & Nov. & Oct. & Sept. & Août & Juil. \\
\hline
\end{tabular}

La comparaison des deux configurations conduit à choisir dans la majorité des cas l'implantation en losange. Si l'on ne recherche pas un fonctionnement saisonnier (en particulier hivernal), elle est en effet le plus souvent préférable. Elle possède de plus une propriété intéressante : pour une valeur de $\tau$ supérieure à environ 3 , celle de $\xi$ est pratiquement indépendante du coefficient de déformation. L'effet de sommation sur les différents mois de l'année est à l'origine de cette propriété que l'on ne retrouve pas pour le coefficient journalier de captage $\xi_{\delta}$, comme le montre le tableau II.

Tableau II. - Configuration en losange, $\tau=3,5$.

$\begin{array}{cccc}\xi_{\delta}(\%) & & \tau^{\prime} & \\ - & 0,7 & 1 & 1,3 \\ \text { Sols. hiver } & 86,65 & 82,58 & 87,37 \\ \text { Equinoxes } & 96,86 & 95,67 & 93,66 \\ \text { Sols. été } & 94,12 & 97,54 & 99,03\end{array}$

Dans un souci de clarification, la configuration en losange sera systématiquement adoptée dans la suite de cette étude. Enfin, les valeurs optimales des coefficients $\tau$ et $\tau^{\prime}$ seront obtenues en dernier lieu sur critères physico-économiques.

2. Etude du réseau de transfert thermique. - Les capteurs, conçus pour fonctionner entre deux niveaux de température prédéterminés, doivent être montés en parallèle entre les canalisations à basse température (avant échauffement) et les canalisations à haute température. Pour une centrale de type THEK, ce sont des vannes de régulation qui asservissent la température du thermofluide en sortie de chaudière.

Si l'ensoleillement est uniformément réparti sur le terrain, un débit pratiquement identique de fluide caloporteur dans chacun des capteurs est ainsi obtenu. Ces derniers doivent être connectés de telle manière que les différents éléments, puissance de pompage, pertes thermiques en ligne, inertie thermique et coût du système, se trouvent minimisés. Le mode de connexion proposé est présenté figure 5.

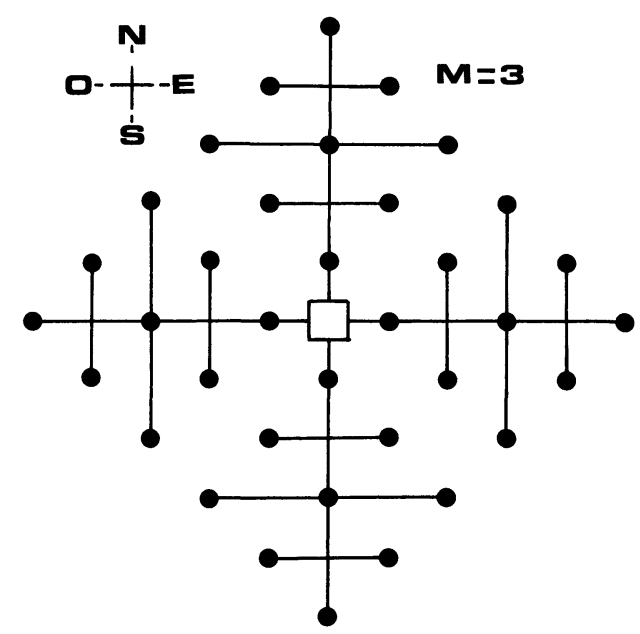

Fig. 5. - Mode de connexion proposé ; les capteurs sont schématisés par des points, les doubles canalisations par des traits et le carré central représente le convertisseur énergétique. Chacun des quatre collecteurs alimente $(M-1)^{2}$ éléments de branches dont $(4(M-2 r)+\varepsilon)$ de débit égal à $r$ fois le débit unitaire, avec, $\varepsilon=1$ si $M=2 r$ et $\varepsilon=0$ dans les autres cas.

[Schematic of the fluid transport network; collectors are figured by points, double pipes by lines and the central production unit by a square. Each of the four collecting lines is connected with $(M-1)^{2}$ branch elements; the flow rate is $r$ times the flow rate unit in $(4(M-2 r)+\varepsilon)$ branch elements with $\varepsilon=1$ if $M=2 r$ and $\varepsilon=0$ in other cases.]

Le convertisseur, thermique ou thermo-électrique, occupe une position centrale, de manière, en particulier, à réduire les pertes de charge du réseau. $\mathrm{Ce}$ dernier offre par ailleurs l'avantage de présenter une double symétrie : une bonne répartition du fluide sur le terrain, ainsi qu'une minimisation des plus gros diamètres de tuyaux sont évidemment favorisées par la présence de quatre collecteurs. Les travaux menés sur différents modes de connexion ont montré que ce type de réseau conduisait à des longueurs de canalisation et à des masses thermiques (de fluide, de tuyaux et d'isolant) relativement faibles. De tels résultats ont une incidence immédiate sur les quantités à minimiser citées plus haut.

Le programme numérique mis au point permet l'étude du réseau de transfert thermique qui vient d'être défini. Il détermine, en premier lieu, les canalisations normalisées à employer, ainsi que les épaisseurs d'isolant adéquates, ceci, pour chacun des tronçons du réseau de transfert. En second lieu, il conduit au calcul de la puissance de pompage, des pertes thermiques en ligne, de l'inertie thermique en fonction du temps de non-fonctionnement, enfin, du coût.

Notre étude est menée pour des centrales solaires dont le nombre d'héliostats est de la forme $4 \mathrm{M}^{2}$. Les données suivantes sont nécessaires au calcul numérique :

- flux solaire incident $(H)$,

- rendement de conversion héliothermique $\left(\eta_{\mathrm{c}}\right)$, 
- surface de captage $(S)$,

- température ambiante $\left(T_{\mathrm{a}}\right)$, niveaux de température haut $\left(T_{\mathrm{h}}\right)$ et bas $\left(T_{\mathrm{b}}\right)$ dans les canalisations,

- pentes de variation avec la température et valeurs à $0{ }^{\circ} \mathrm{C}$ de la masse volumique $(\rho)$ et de la chaleur spécifique $(C)$, du fluide caloporteur, ainsi que les valeurs des viscosités de ce fluide aux températures haute et basse,

- gamme des diamètres normalisés, extérieurs et intérieurs des canalisations, masse volumique et chaleur spécifique du matériau les constituant,

- pente de variation avec la température et valeur à $0 \quad{ }^{\circ} \mathrm{C}$ de la conductivité thermique de l'isolant, de sa capacité calorifique et de sa masse volumique, ainsi que le coefficient d'isolation $(A)$ défini plus loin,

- coûts de l'unité de volume du fluide caloporteur $\left(F_{\mathrm{f}}\right)$, des tuyaux $\left(F_{\mathrm{t}}\right)$, et de l'isolant $\left(F_{\mathrm{i}}\right)$ posés,

- vitesse théorique (faisant abstraction de toute normalisation) en chaudière $\left(C_{\mathrm{c}}\right)$ et dans les canalisations n'alimentant qu'un seul convertisseur héliothermique $\left(C_{1}\right)$, pour l'ensoleillement nominal

$H=800 \mathrm{~W} / \mathrm{m}^{2}$.

C'est cette dernière donnée qui permet la détermination des diverses canalisations à employer ; nous avons en effet opté pour un écoulement à perte de charge linéique constante dans le réseau. L'avantage d'un tel type d'écoulement, par rapport, par exemple, à un écoulement à vitesse constante, est indéniable, notamment en ce qui concerne l'inertie thermique et le coût du réseau de transfert. Dans l'hypothèse envisagée, les plus faibles vitesses de circulation sont obtenues pour les plus petits diamètres de tuyaux et inversement.

Après le calcul du débit massique en chaudière, ou unitaire $(Q)$, d'après :

$Q \int_{T_{\mathrm{b}}}^{T_{\mathrm{h}}} C \mathrm{~d} T=\eta_{\mathrm{c}} H S$

on obtient la perte de charge de circulation, soit, la perte de charge dans la canalisation non normalisée où le fluide circule avec un débit $Q$ à la vitesse $C_{1}$.
Chaque tronçon de canalisation, dans lequel le débit est évidemment égal à un multiple de $Q$, est ensuite déterminé par le calcul de la vitesse théorique et le choix de la canalisation normalisée entraînant une vitesse réelle aussi voisine que possible de celle-ci. En chaudière, on se limite au choix de la canalisation, tel que vitesse réelle de circulation et vitesse théorique $C_{\mathrm{c}}$, déterminée par des considérations de bon transfert thermique soient aussi voisines que possible.

C'est le coefficient d'isolation $A$ qui conduit au calcul des épaisseurs d'isolant appropriées. En supposant que le coût de l'isolation soit proportionnel au volume d'isolant posé, l'épaisseur propre à chaque tronçon de canalisation est déterminée sur critères économiques d'après l'équation différentielle suivante :

$\frac{\delta p_{l}}{\delta v_{\mathrm{i}}}=A$

$v_{\mathrm{i}}$ est ici le volume d'isolant et $p_{l}$ représente les pertes thermiques en ligne, par unité de longueur de la canalisation considérée (Annexe 1). Cette épaisseur est ensuite normalisée, c'est-à-dire arrondie au multiple d'un centimètre le plus voisin. A titre d'exemple, sont données dans le tableau III les caractéristiques du réseau de transfert d'une centrale comportant 100 capteurs ; les valeurs de $C_{1}, C_{\mathrm{c}}$, et $A$ ont respectivement été prises égales à $0,75 \mathrm{~m} / \mathrm{s}, 2,5 \mathrm{~m} / \mathrm{s}$, et $-1000 \mathrm{~W} / \mathrm{m}^{3}$; celles de $T_{\mathrm{a}}, T_{\mathrm{h}}$ et $T_{\mathrm{b}}$ à $10{ }^{\circ} \mathrm{C}$, $300{ }^{\circ} \mathrm{C}$ et $200^{\circ} \mathrm{C}$. L'isolant est de la laine de verre, le fluide du Gilotherm TH et les tuyaux sont en acier noir de normes NF A 49-115 (faibles diamètres) ou NF A 49-111.

Le réseau de transfert thermique est maintenant défini ; on peut donc aborder l'étude de ses différents éléments caractéristiques.

Le calcul de la puissance de pompage nécessite celui des pertes de charge dans les conduites reliant le convertisseur énergétique aux capteurs les moins accessibles pour le fluide caloporteur. Ils sont théoriquement au nombre de 2 si $\tau^{\prime} \neq 1$ et situé en bout de collecteurs : Est-Ouest si $\tau^{\prime}>1$, Nord-Sud si $\tau^{\prime}<1$. Par ailleurs, la prise en compte des pertes de charge singulières, pratiquement réduites à celles

Tableau III

$\begin{array}{lccccccccccc}\quad \text { Débit } & 1 Q & 2 Q & 3 Q & 6 Q & 10 Q & 15 Q & 19 Q & 22 Q & 24 Q & 25 Q & \begin{array}{c}Q \\ \text { Chaudière }\end{array} \\ \begin{array}{l}\text { Dénomination } \\ \text { tube (1) }\end{array} & 15 \times 21 & 20 \times 27 & 20 \times 27 & 26 \times 34 & 33 \times 42 & 40 \times 49 & 49 \times 54 & 49 \times 54 & 51 \times 57 & 51 \times 57 & 8 \times 13 \\ \begin{array}{l}\text { Ep. isolant } \\ \text { (cm) } 300^{\circ} \mathrm{C}\end{array} & 6 & 7 & 7 & 7 & 7 & 8 & 8 & 8 & 8 & 8 & 6 \\ \begin{array}{l}\text { Ep. isolant } \\ \text { (cm) } 2000^{\circ} \mathrm{C}\end{array} & 5 & 5 & 5 & 6 & 6 & 6 & 6 & 6 & 6 & 6 & \\ \begin{array}{c}\text { Vitesse moy. } \\ (\mathrm{m} / \mathrm{s}) 300-200\end{array} & 0,68 & 0,75 & 1,13 & 1,43 & 1,37 & 1,51 & 1,41 & 1,64 & 1,62 & 1,69 & 2,24\end{array}$

( ${ }^{1}$ ) Les dimensions exactes des tubes sont légèrement différentes de celles apparaissant dans leur dénomination. 
provoquées par les vannes de régulation, s'effectue en supposant ces dernières ouvertes pour un flux solaire incident direct $\left(1 \mathrm{~kW} / \mathrm{m}^{2}\right)$ supérieur aux maxima réels. Dans tous les cas envisagés, le régime de circulation est turbulent rugueux. Le coefficient de rugosité des conduites a été supposé nul et le rendement du système de pompage constant. Le terme puissance de pompage, exprimé par capteur, peut alors se mettre sous la forme linéaire :

$P_{\mathrm{p}}=P_{\mathrm{pc}} \operatorname{Max}\left(L_{1}, L_{2}\right)+P_{\mathrm{p} 3} L_{3}+P_{\mathrm{p} 4} L_{4}$

où $L_{1}$ et $L_{2}$ sont les longueurs des diagonales du losange d'implantation selon les directions Est-Ouest et Nord-Sud, $L_{3}$ est la longueur de canalisation du bas du capteur à la chaudière, $L_{4}$ la longueur du tube chaudière.

On calcule les valeurs des coefficients de linéarisation $\boldsymbol{P}_{\mathrm{pc}}, \boldsymbol{P}_{\mathrm{p} 3}$ et $\boldsymbol{P}_{\mathrm{p} 4}$ pour un dimensionnement quelconque de la centrale; par interpolation, on peut en déduire le graphe de la figure 6 . Les variations de ces coefficients y sont données pour un nombre $(n)$ d'héliostats compris entre 4 et 100000 , un rendement du système de pompage égal à 0,85 , une puissance solaire incidente de $1000 \mathrm{~W} / \mathrm{m}^{2}$. Les autres hypothèses sont celles correspondant au tableau III.

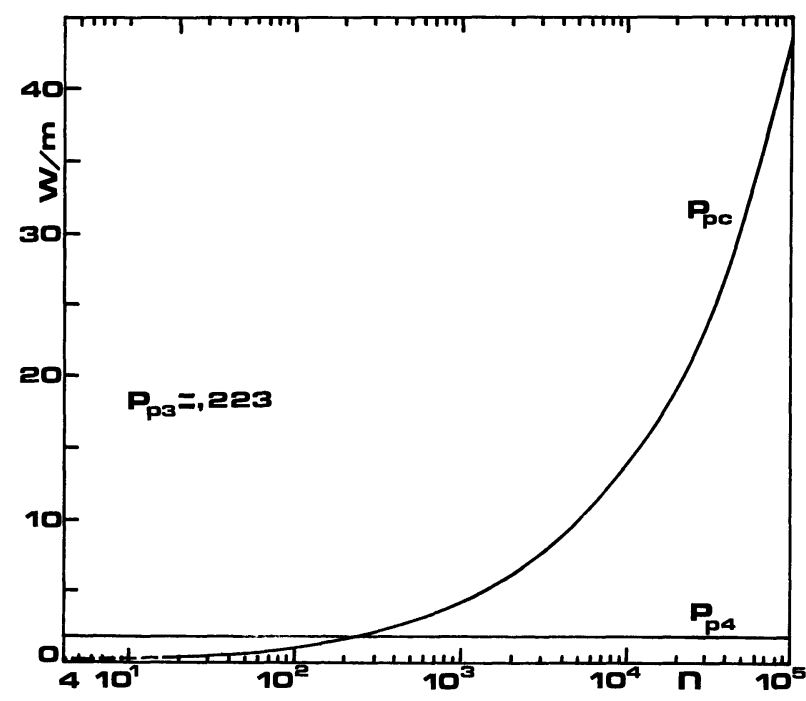

Fig. 6. - Variations, avec le nombre d'héliostats, des coefficients servant à la détermination de la puissance de pompage nécessaire à la circulation du thermofluide.

[Variations of the coefficients used to calculate the pumping power, versus the number of collectors.]

D'une façon similaire à ce qui a été fait pour la puissance de pompage, il est possible de linéariser les pertes thermiques en ligne par rapport aux dimensions caractéristiques du réseau. Dans ce but, nous avons procédé aux approximations suivantes : la résistance de transfert thermique entre le fluide caloporteur et l'air a été réduite à celle de l'isolant seul, et la décroissance de la température du fluide le long des canalisations a été négligée. Les vitesses de circulation étant relativement élevées et les épaisseurs d'isolant importantes, on a vérifié la quasiexactitude des résultats obtenus. Les pertes thermiques en ligne de l'ensemble du réseau de transfert, rapportées à un capteur, peuvent alors s'écrire :

$P_{l}=\left(P_{l \mathrm{c}}+P_{l \mathrm{~b}}\right)\left(L_{1}+L_{2}\right)+P_{l 3} L_{3}$.

Les coefficients $P_{l \mathrm{c}}$ et $P_{l \mathrm{~b}}$ traduisent les contributions à $P_{l}$, respectivement, des collecteurs et des branches du réseau. Les courbes de variation des coefficients $P_{l \mathrm{c}}, P_{l \mathrm{~b}}$ et $P_{l 3}$, en fonction du nombre d'héliostats, sont représentées figure 7 .

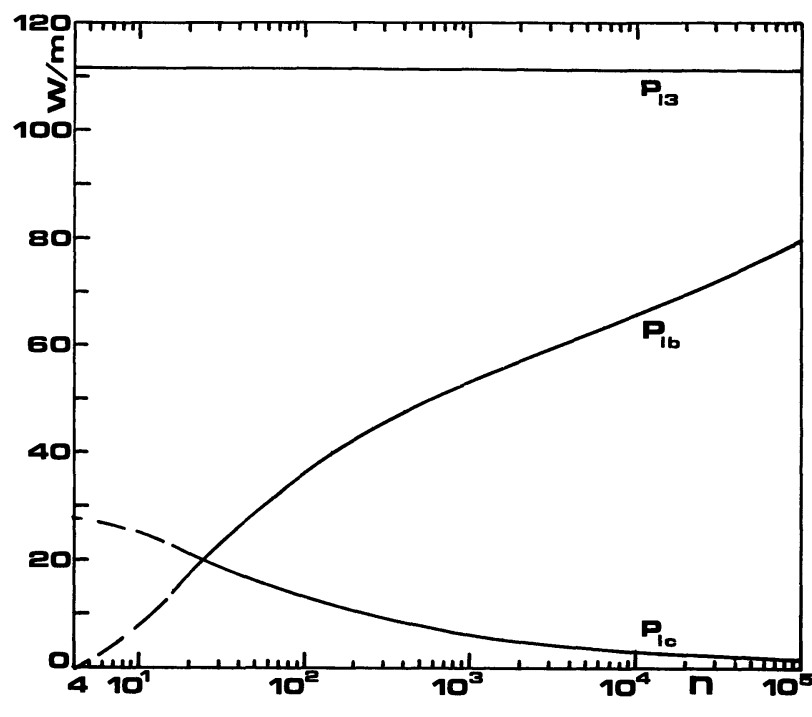

Fig. 7. - Variations, avec le nombre d'héliostats, des coefficients servant à la détermination des pertes thermiques en ligne.

[Variations of the coefficients used to calculate network thermal losses, versus the number of collectors.]

L'inertie thermique $\left(W_{\mathrm{t}}\right)$ du réseau de transfert peut être définie comme la quantité d'énergie à fournir à l'ensemble, fluide, tube et isolant, pour le ramener à son état initial, supposé être celui d'un fonctionnement normal (niveaux de température hauts et bas établis), après un temps d'interruption déterminé $(t)$ de l'ensoleillement. Le terme $W_{t}$, par capteur, est évidemment proportionnel aux dimensions caractéristiques du système :

$$
\begin{gathered}
W_{\mathrm{t}}=\left(W_{\mathrm{tc}}+W_{\mathrm{tb}}\right)\left(L_{1}+L_{2}\right)+ \\
+W_{\mathrm{t} 3} L_{3}+W_{\mathrm{t} 4} L_{4}
\end{gathered}
$$

Les calculs de $W_{\mathrm{tc}}, W_{\mathrm{tb}}, W_{\mathrm{t} 3}$ et $W_{\mathrm{t} 4}$ sont effectués pour diverses valeurs de $t$.

L'étude du refroidissement des canalisations tient compte de la masse thermique induite par la présence de l'isolant et des variations, avec la température, des paramètres caractéristiques ( $\rho$ et $C$ ) du fluide 
caloporteur et de la conductivité thermique de l'isolant ; elle suppose que ce refroidissement s'effectue selon une superposition de régimes permanents et que, comme pour les pertes thermiques en ligne, la résistance de transfert fluide-air est égale à celle due à l'isolant seul. Avec les mêmes hypothèses que pour le tableau III, sont données dans le tableau IV les températures finales du fluide, dans les canalisations d'une centrale de 100 capteurs, pour quatre valeurs du temps d'interruption $(t)$.

Tableau IV

$\begin{array}{cccccccc}T_{\mathrm{h}} / T_{\mathrm{b}}\left({ }^{\circ} \mathrm{C}\right) & 1 Q & 2 Q & 6 Q & 10 Q & 15 Q & 19 Q & 24 Q \\ - & - & - & - & - & - & - & - \\ 45 \text { minutes } & 185 / 125 & 213 / 139 & 232 / 157 & 246 / 165 & 256 / 170 & 260 / 173 & 263 / 174 \\ 3 \text { heures } & 63 / 43 & 93 / 57 & 121 / 83 & 146 / 100 & 168 / 109 & 177 / 115 & 184 / 119 \\ 12 \text { heures } & 11 / 10 & 15 / 11 & 22 / 17 & 33 / 23 & 46 / 29 & 53 / 32 & 59 / 36 \\ 24 \text { heures } & 10 / 10 & 10 / 10 & 10 / 10 & 13 / 11 & 16 / 12 & 19 / 13 & 21 / 14\end{array}$

Remarquons qu'à l'énergie $W_{t}$, on peut associer le terme $W_{\mathrm{t}} / \eta_{\mathrm{c}} S H_{\mathrm{e}}$ qui est le temps de fonctionnement perdu par capteur pour une puissance solaire incidente captée $H_{\mathrm{e}}$. On peut également y associer le terme $W_{\mathrm{t}} / t$, l'équivalent pertes thermiques en ligne à $W_{\mathrm{t}}$, pendant le temps d'interruption.

Sur la figure 8 apparaissent les variations de $W_{\text {tc }}$, $W_{\mathrm{tb}}, W_{\mathrm{t} 3}$ et $W_{\mathrm{t} 4}$ pour $t=12$ heures et $90 \mathrm{~min}$.

Dans le calcul du coût, on suppose les prix du fluide, des tuyaux et d'isolant posés proportionnels à leur volume. Le coût du réseau de transfert, par capteur, peut se mettre sous la forme linéaire :

$C=\left(C_{\mathrm{c}}+C_{\mathrm{b}}\right)\left(L_{1}+L_{2}\right)+C_{3} L_{3}$

Les variations des différents coefficients $C_{\mathrm{c}}, C_{\mathrm{b}}$ et $C_{3}$ apparaissent figure 9 ; les hypothèses émises sont précisées dans la légende.

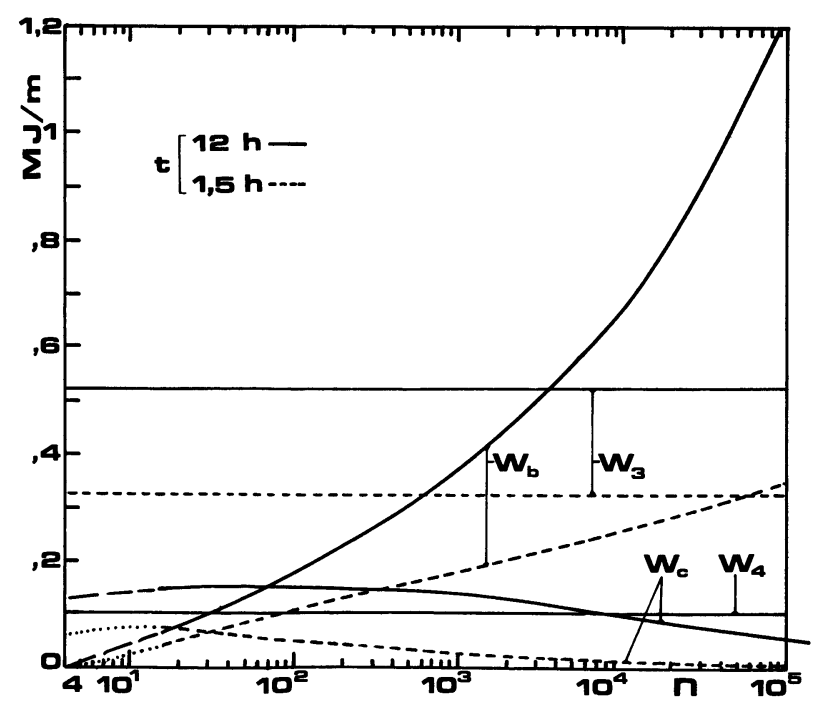

Fig. 8. - Variations, avec le nombre d'héliostats, et pour deux valeurs du temps d'interruption, des coefficients servant à la détermination de l'inertie thermique du réseau de transfert.

[Variations, for two interruption periods, of the coefficients used to calculate the network thermal inertia, versus the number of collectors.]

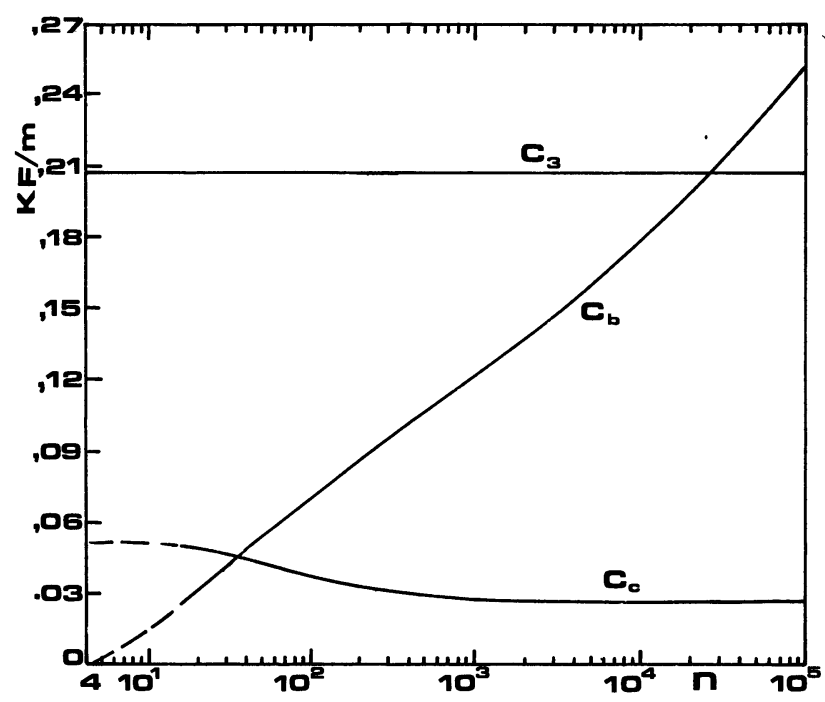

Fig. 9. - Variations, avec le nombre d'héliostats, des coefficients servant à la détermination du coût du réseau de transfert ; les données nécessaires au calcul sont : $F_{\mathrm{t}}=150 \mathrm{kF} / \mathrm{m}^{3}, F_{\mathrm{i}}=6 \mathrm{kF} / \mathrm{m}^{3}$, $F_{\mathrm{f}}=6 \mathrm{kF} / \mathrm{m}^{3}$.

[Variations of the coefficients used to calculate the network cost, versus the number of collectors; economical parameters are : $F_{\mathrm{t}}=150 \mathrm{kF} / \mathrm{m}^{3}, F_{\mathrm{i}}=6 \mathrm{kF} / \mathrm{m}^{3}, F_{\mathrm{f}}=6 \mathrm{kF} / \mathrm{m}^{3}$.]

Quelles que soient les dimensions caractéristiques des centrales solaires étudiées, les éléments, puissance de pompage, pertes thermiques en ligne, inertie thermique et coût sont maintenant aisément calculables. Dans le paragraphe suivant, l'étude du réseau de transfert est connectée à celle du premier paragraphe, dans le but de l'optimisation de l'implantation des capteurs sur le terrain.

3. Optimisation du réseau. - Il s'agit ici d'élaborer une méthode de calcul qui permette l'optimisation du réseau de transfert thermique des centrales solaires à convertisseurs distribués, puis, d'appliquer cette 
méthode aux capteurs de type THEK. Il faut, pour le mode de connexion adopté, calculer les distances $L_{1}$ et $L_{2}$ conduisant aux systèmes les plus performants, ou encore, déterminer les valeurs optimales des coefficients d'espacement $(\tau)$ et de déformation $\left(\tau^{\prime}\right)$.

On peut, en premier lieu, montrer qu'indépen.damment de la valeur du coefficient $\tau$, les valeurs les plus adéquates de $\tau^{\prime}$ sont voisines de 1 .

Les grandeurs, pertes thermiques et coût du réseau de transfert, croissent en effet linéairement avec les longueurs de canalisations au sol, elles-mêmes proportionnelles à $\left(L_{1}+L_{2}\right)$, or :

$L_{1}+L_{2}=\sqrt{2} R \tau\left(\tau^{\prime}+1 / \tau^{\prime}\right)$.

La puissance électrique nécessaire au système de pompage, ainsi que son coût, augmentent avec $\operatorname{Max}\left(L_{1}, L_{2}\right)$ et l'on a :

$$
\begin{aligned}
& \operatorname{Max}\left(L_{1}, L_{2}\right)=\sqrt{2} R \tau \tau^{\prime} \quad \text { si } \quad \tau^{\prime}>1 \\
& =\sqrt{2} R \tau / \tau^{\prime} \quad \text { si } \tau^{\prime}<1 .
\end{aligned}
$$

D'après les relations ci-dessus, $\left(L_{1}+L_{2}\right)$ et Max $\left(L_{1}, L_{2}\right)$ présentent des minima pour une valeur de $\tau^{\prime}=1$; c'est donc pour cette valeur que les grandeurs étudiées dans le paragraphe précédent se trouvent minimisées.

D'autre part, d'après le graphe de la figure 4, on a déjà remarqué que pour l'implantation en losange, le coefficient annuel de captage n'était que faiblement sensible aux variations du coefficient de déformation.

Il apparaît ainsi que la valeur appropriée de $\tau^{\prime}$ est approximativement égale à 1 . Par ailleurs, si l'on ne prend pas en considération le coût du terrain, il est encore possible de vérifier qu'à une implantation $\left(\tau, \tau^{\prime}\right)$ définie, correspond l'implantation $\left(\tau_{1}, 1\right)$ conduisant aux mêmes longueurs de canalisation, et pour laquelle la valeur du coefficient annuel de captage est plus élevée. Dans la suite de ce travail, on adoptera systématiquement 1 pour valeur optimale de $\tau^{\prime}$.

On peut maintenant envisager l'optimisation du coefficient d'espacement. Celle-ci doit s'effectuer suivant un critère économique ; ainsi, le système sera considéré comme optimisé lorsque la valeur du bénéfice net actualisé sera maximale au cours de l'exploitation de la centrale. Ce bénéfice net actualisé $(B)$ dépend de plusieurs paramètres dont l'investissement initial $(I)$, le prix de vente de l'énergie $(F)$, la quantité d'énergie produite par an $(x)$, et le montant de la dépense d'entretien $(D)$. D'une manière classique, $B$ s'écrit :

$B=-I+(F x-D) S_{\alpha, \beta}$

où $S_{\alpha, \beta}$ est une fonction du taux d'intérêt de l'argent $(\alpha)$ et du délai de récupération du capital $(\beta)$ :

$S_{\alpha, \beta}=\frac{1}{\alpha}\left(1-\frac{1}{(1+\alpha)^{\beta}}\right)$.
Ces différents paramètres dépendent de multiples variables, dont $\tau$; c'est l'annulation de la dérivée partielle de $\mathrm{B}$ par rapport à $\tau$ qui conduit à l'équation différentielle que doit vérifier la valeur optimale du coefficient d'espacement. Si l'on néglige l'éventuelle dépendance de la dépense annuelle d'entretien avec $\tau$, il vient :

$\frac{\delta x}{\delta \tau}=\frac{\delta I}{\delta \tau} \frac{1}{F S_{\alpha, \beta}}$.

Dans cette égalité apparaît clairement le caractère physico-économique du problème posé. On peut, tout d'abord, en étudier la partie strictement physique, soit, le premier membre de la relation (16).

Si l'on néglige les variations de la consommation électrique du système de pompage avec le débit $\mathrm{du}$ thermofluide, en supposant notamment sa puissance non adaptable au rayonnement incident, l'énergie produite sous forme thermique $\left(x_{t}\right)$, par capteur, pendant le temps de fonctionnement annuel $\left(t_{\mathrm{f}}\right)$, peut s'écrire :

$x_{\mathrm{t}}=S \overline{\bar{H}} \eta_{\mathrm{c}} \xi-P_{\mathrm{l}} t_{\mathrm{f}}-e P_{\mathrm{p}} t_{\mathrm{f}}-W_{\mathrm{i}}$

$\overline{\bar{H}}$ représente la quantité d'énergie récupérable par an et $W_{\mathrm{i}}$ celle annuellement perdue du fait de l'inertie thermique du réseau de transfert. Le paramètre e est un coefficient de pondération, l'énergie nécessaire au système de pompage étant de nature électrique et non pas thermique. On le prendra égal à l'inverse $\mathrm{du}$ rendement de conversion thermo-électrique $\left(\eta_{\mathrm{e}}\right)$, si l'installation est productrice d'électricité ; si elle est productrice de chaleur, on pourra le prendre égal au rapport du prix de vente de l'énergie électrique à celui de l'énergie sous forme thermique. La valeur de $x$ définie dans (14) se déduit aisément de $x_{\mathrm{t}}$ :

- dans le cas d'une centrale productrice de chaleur : $x=x_{1}$

- dans le cas d'une centrale productrice d'électricité : $x=\eta_{\mathrm{e}} x_{\mathbf{t}}$.

Nous nous plaçons ci-dessous dans la seconde de ces hypothèses. Les expressions correspondant à la première se déduiront simplement de celles obtenues, en égalant à 1 le rendement de conversion thermo-électrique.

Il est maintenant nécessaire de déterminer les valeurs de $t_{\mathrm{f}}$ et de $W_{\mathrm{i}}$. Dans ce but, nous avons formulé les hypothèses simplificatrices suivantes :

- le rayonnement de seuil de la centrale $\left(H_{\mathrm{s}}\right)$ est indépendant de son dimensionnement,

- une journée de non-fonctionnement de cette centrale ou mauvais jour est celle où la durée d'insolation n'a pas excédé $m$ unité de temps, quelle que soit leur répartition, continue ou discontinue notamment, au cours de la journée,

- la probabilité $p$ d'avoir, au lendemain d'un jour donné, un mauvais jour, s'effectue sans corré- 
lation d'ordre météorologique [4]. Si $N$ est le nombre de mauvais jours par an, $p$ est donc égal à $N / 365$. Pour la même raison, la probabilité d'avoir une séquence de $i$ mauvais jours est égale à $p^{i}(1-p)$,

- le temps de non-fonctionnement correspondant aux passages nuageux se partage également entre occultations de diverses durées : l'occultation, que l'on suppose non consécutive à une nuit, est caractérisée par sa durée ; à chaque durée envisagée correspondra annuellement le même temps de non-insolation

Il a été procédé de la façon suivante : après l'évaluation raisonnable des valeurs de $m$ et $H_{s}$, on a déterminé, d'après les relevés effectués à Carpentras [3], l'énergie maximale récupérable par an $\left(\overline{\bar{H}}_{\mathrm{s}}\right)$, le temps maximal de fonctionnement $\left(t_{\mathrm{s}}\right)$, ainsi que le nombre de mauvais jours $(N)$. De la valeur de $t_{\mathrm{s}}$ il résulte le temps minimal de non-fonctionnement $\left(t_{\mathrm{ns}}\right)$. Par ailleurs, du fait de la dernière hypothèse simplificatrice, la durée d'ensoleillement d'un mauvais jour est égale à $m / 2$. On peut alors en déduire le temps de fonctionnement de la centrale $\left(t_{\mathrm{f}}\right)$ et son temps de nonfonctionnement $\left(t_{\mathrm{nf}}\right)$, ainsi que l'énergie récupérable $(\overline{\bar{H}})$, d'après :

$t_{\mathrm{f}}=t_{\mathrm{s}}-N \mathrm{~m} / 2$

$t_{\text {nf }}=t_{\text {ns }}+N m / 2$

$\overline{\bar{H}}=\overline{\overline{H_{\mathrm{s}}}} t_{\mathrm{f}} / t_{\mathrm{s}}$.

Pour le calcul de $W_{\mathrm{i}}$, il faut distribuer le temps de non-fonctionnement $t_{\mathrm{nf}}$ entre nuits, mauvais jours et passages nuageux. Il est possible d'approximer une telle distribution en raisonnant sur des journées de type équinoxe. Les parts de $t_{\text {nf }}$ correspondant aux nuits et aux mauvais jours étant ainsi déterminées, on en déduit celle réservée aux passages nuageux $\left(t_{\mathrm{n}}\right)$ ayant lieu pendant les jours de fonctionnement.

Après avoir vérifié que $H_{\mathrm{s}}$ variait faiblement avec $n$, nous avons posé :

$H_{\mathrm{s}} \simeq 110 \mathrm{~W} / \mathrm{m}^{2}$ et $m=2$ heures

et nous avons ainsi obtenu :

$\overline{\bar{H}}_{\mathrm{s}}=1663, \quad \overline{\bar{H}}=1629(\mathrm{kWh})$

$N=56$ (jours)

$t_{\mathrm{f}}=2719, \quad t_{\mathrm{nf}}=6041, \quad t_{\mathrm{n}}=989$ (heures).

Du fait des hypothèses émises, il vient, pour expression de $W_{\mathrm{i}}$ (Annexe II) :

$$
\begin{aligned}
W_{\mathrm{i}}= & \sum_{j=0}^{j=\infty} N_{j} W_{\mathrm{t}=(2 j+1) 12 \text { heures }}+ \\
& +\frac{t_{\mathrm{n}}}{12-m} \int_{0}^{(12-m) \text { heures }} \frac{W_{\mathrm{t}}}{t} \mathrm{~d} t
\end{aligned}
$$

où $(12-m)$ heures est la durée maximale d'un nuage au cours d'une journée de fonctionnement et $N_{j}$. Si $j \neq 0$ le nombre de séquences par année de $j$ mauvais jours consécutifs.

$$
\begin{aligned}
\text { Si } j=0, \quad N_{j} & =365(1-p)^{2} p^{j} . \\
N_{0} & =365-N(2-p) .
\end{aligned}
$$

Il est maintenant possible d'exprimer le terme $\delta x / \delta \tau$; les termes $P_{l} t_{\mathrm{f}}$, e $P_{\mathrm{p}} t_{\mathrm{f}}$ et $W_{\mathrm{i}}$ apparaissant dans (17) varient linéairement avec $\tau$; on peut donc écrire :

$$
\frac{\delta x}{\delta \tau}=S \overline{\bar{H}} \eta_{\mathrm{c}} \eta_{\mathrm{e}}\left(\frac{\delta \xi}{\delta \tau}-C_{n}\right)
$$

où $C_{n}$ ne dépend pas de $\tau$ mais du nombre d'héliostats (n) de la centrale. Si le coefficient d'espacement devient important, on a la relation suivante :

$\frac{\delta x}{\delta \tau} \simeq-S \overline{\bar{H}} \eta_{\mathrm{c}} \eta_{\mathrm{e}} C_{n}$

les capteurs sont alors suffisamment éloignés pour que n'intervienne plus le terme dû aux effets d'ombre.

Sur le graphe de la figure 10 sont portées les variations de $\delta \xi / \delta \tau$ en fonction de $\tau$; elles ont été obtenues pour la configuration en losange et pour $\tau^{\prime}=1$.

Sur la figure 11, apparaissent les variations de $C_{n}$ en fonction du nombre de capteurs de la centrale. Les diverses contributions à $C_{n}$ des éléments physiques pris en considération ont de plus été mises en évidence. Pour les plus faibles nombres de capteurs, les pertes thermiques en ligne constituent le para-

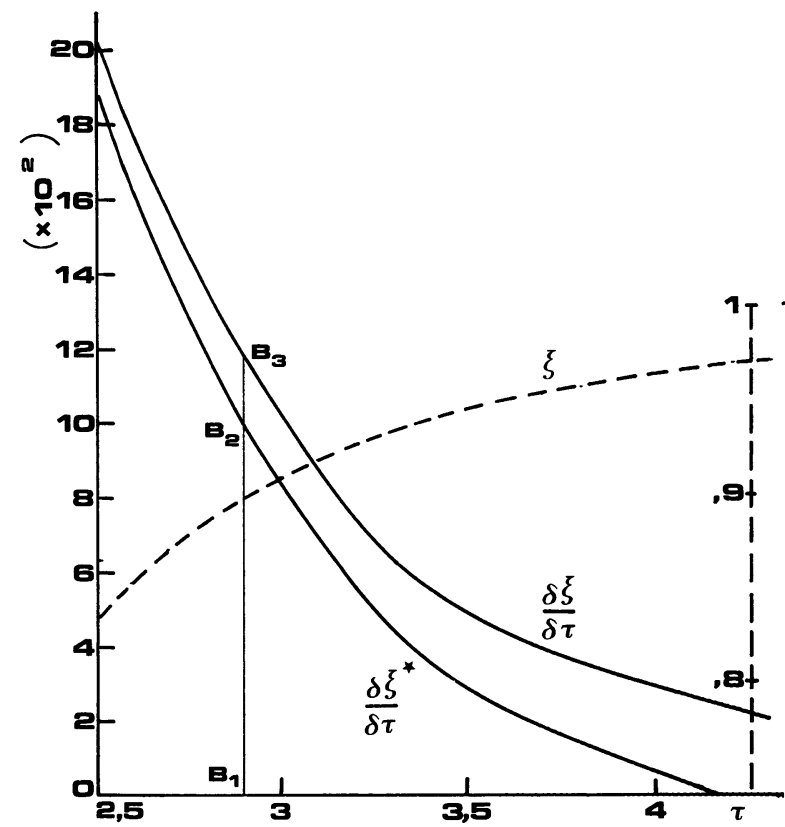

Fig. 10. - Variations, avec le coefficient d'espacement, des grandeurs $\delta \xi / \delta \tau$ et $(\delta \xi / \delta \tau)^{*}$, conduisant à l'optimisation du réseau de transfert thermique sur critères physiques ou physico-économiques. En pointillés, on a représenté la courbe $\xi(\tau)$.

[Variations, versus the spacing coefficient, of $\partial \xi / \partial \tau$ and $(\partial \xi / \partial \tau)^{*}$ used to optimize the fluid transport network, according to physical or physical and economical parameters. $\xi(\tau)$ is given in broken line.] 


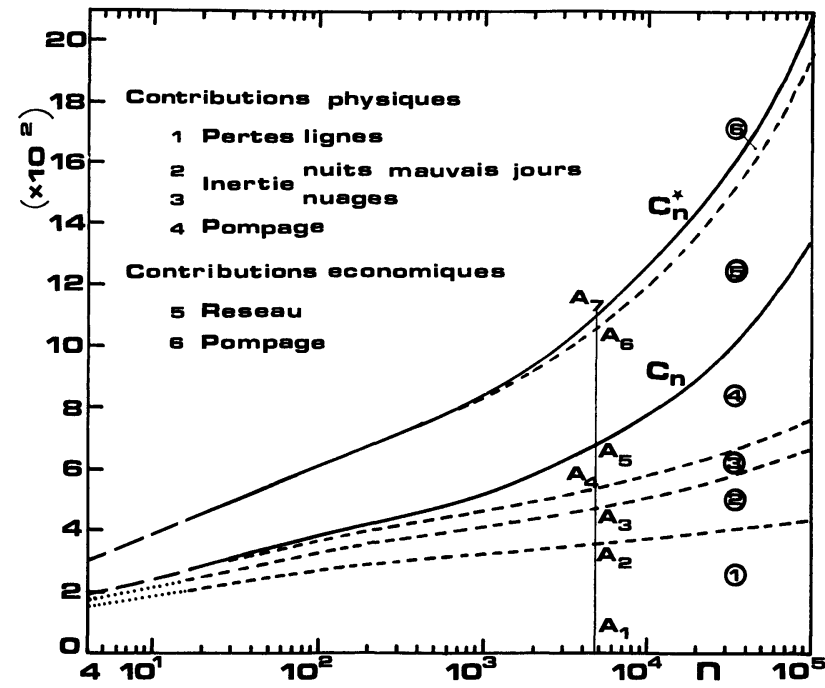

Fig. 11. - Variations, avec le nombre d'héliostats, des grandeurs $C_{n}$ et $C_{n}^{*}$, nécessaires à l'optimisation du réseau de transfert sur critères physiques ou physico-économiques. A chaque élément considéré correspond sur le graphe une région représentant la contribution à $C_{n}$ ou à $C_{n}^{*}$ de cet élément.

[Variations, versus the number of collectors, of $C_{n}$ and $C_{n}^{*}$ used to optimize the fluid transport network, according to physical or physical and economical parameters. To each factor corresponds, on the graph, an area which represents its contribution to $C_{n}$ or $C_{n}^{*}$.]

mètre prépondérant. Lorsque ce nombre devient important, aucun des quatre facteurs considérés ne peut plus être négligé. Les variations sensibles de $C_{n}$ sont alors dues à la part croissante prise par la puissance de pompage. Celle-ci a été calculée avec une valeur de e égale à 5 . Remarquons que dans tous les cas, l'élément inertie thermique reste relativement faible; le résultat n'est évidemment pas semblable quand le réseau étudié utilise une vitesse de circulation du fluide constante, de l'ordre de 1 à $1,5 \mathrm{~m} / \mathrm{s}$.

L'optimisation du système sur critères exclusivement physiques, c'est-à-dire en supposant un investissement indépendant de $\tau$, est maintenant envisageable. C'est l'équation suivante qui y conduit :

$\frac{\delta \xi}{\delta \tau}-C_{n}=0$.

Dans le tableau $\mathrm{V}$ sont données les valeurs adéquates du coefficient d'espacement pour des centrales de divers dimensionnements.

Tableau V

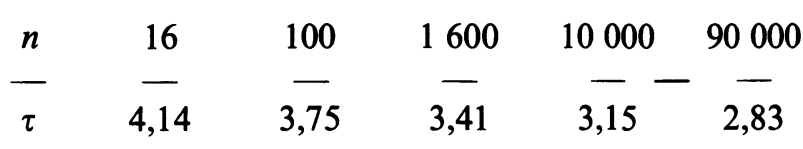

L'étude de l'incidence économique sur les résultats obtenus ne présente pas de difficultés majeures. Elle conduit à une équation comparable à (26) ; comme nous allons le voir, nous l'écrirons : $\left(\frac{\delta \xi}{\delta \tau}\right)^{*}-C_{n}^{*}=0$.

Pour un dimensionnement déterminé de la centrale, ce sont le coût du réseau de transfert, celui du système de pompage que nous supposerons proportionnel à sa puissance, ainsi que le coût du terrain qui sont à prendre en considération. Les deux premiers de ces éléments varient linéairement avec $\tau$ et les coefficients de linéarisation sont fonction du nombre d'héliostats de la centrale. Le coût du terrain est, pour sa part, proportionnel à sa surface ; celle-ci, par capteur, est égale à $\tau^{2} R^{2}$.

D'après les relations (16) et (24), il vient :

$$
\frac{\delta \xi}{\delta \tau}-C_{n}=\frac{1}{F S_{\alpha, \beta} S \overline{\bar{H}} \eta_{\mathrm{c}} \eta_{\mathrm{e}}} \frac{\delta I}{\delta \tau}
$$

Compte tenu des remarques précédentes, cette relation peut se mettre sous la forme :

$\frac{\delta \xi}{\delta \tau}-C_{n}=C_{n}^{\prime}+f \tau$

De la même manière que $C_{n}, C_{n}^{\prime}$ n'est pas fonction de $\tau$, mais du nombre de capteurs de la centrale, tandis que $f$ ne dépend d'aucun de ces deux paramètres. Par ailleurs, $C_{n}^{\prime}$ et $f$ sont inversement proportionnels au produit $F S_{\alpha, \beta} \eta_{\mathrm{e}}$. Ci-dessous, nous posons ce produit égal à 1 , de telle façon que, dans une hypothèse différente, la valeur exacte de $C_{n}^{\prime}$ puisse être aisément obtenue. Pour une raison similaire, le coût par mètre carré du terrain, auquel est proportionnel $f$, sera pris égal à $10 \mathrm{~F}$.

On est évidemment conduit à la relation (27) en posant :

$\left(\frac{\delta \xi}{\delta \tau}\right)^{*}=\frac{\delta \xi}{\delta \tau}-f \tau$

et

$C_{n}^{*}=C_{n}+C_{n}^{\prime}$.

Les variations de $(\delta \xi / \delta \tau)^{*}$ sont données figure 10 . Remarquons que l'impact du coût du terrain sur le dimensionnement de la maille est le plus important pour les grandes valeurs du coefficient d'espacement.

Sur le graphe de la figure 11 sont portées les variations de $C_{n}^{*}$. De la même manière que pour $C_{n}$, nous avons mis en évidence les contributions à $C_{n}^{*}$ de chacun des éléments économiques considérés. Celle correspondant au système de pompage apparaît dans tous les cas négligeable, ceci, malgré l'hypothèse pessimiste qui a consisté à supposer le coût du $\mathrm{kWe}$ installé indépendant du dimensionnement de ce système et égal à $3000 \mathrm{~F}$. Au contraire, la part correspondant au coût du réseau est relativement importante, en particulier, pour les plus faibles nombres d'héliostats.

Nous sommes finalement conduits à énoncer : l'incidence économique, pour l'optimisation du réseau 
de transfert thermique des centrales solaires à convertisseurs distribués, n'est jamais négligeable, et est d'autant plus importante que cette centrale est petite.

Dans le cas de centrales productrices d'électricité, le paramètre $\eta_{\mathrm{e}}$ est différent de 1 et doit être considéré comme variable avec le dimensionnement de ces centrales. L'impact économique est alors d'autant plus important que les unités de production sont petites. Ce n'est en effet qu'à moyennes et grandes puissances que les rendements de conversion thermoélectrique peuvent, en l'état actuel des techniques, être considérés satisfaisants.

A titre d'exemple, pour des centrales de divers dimensionnements, on envisage ci-dessous trois types d'exploitation. Pour le premier, le coefficient de pondération e, défini d'après (17), a été pris égal à 5 ; pour les deux autres, à l'inverse du rendement $\eta_{\mathrm{e}}$.

1) Une production d'énergie sous forme thermique conduisant à une valeur égale à 1 du produit $F S_{\alpha, \beta}$, soit, par exemple :

$F \simeq 0,12 \mathrm{~F} / \mathrm{kW}, \quad \alpha=10 \%, \quad \beta=20$ ans

2) Une production d'énergie sous forme électrique avec une valeur différente de $\eta_{e}$ selon le nombre d'héliostats envisagé, un prix de vente du $\mathrm{kWe}$ égal à $0,6 \mathrm{~F}$ et des valeurs de $\alpha$ et $\beta$ inchangées par rapport au cas précédent.

3) Une production d'énergie sous forme électrique suivant les hypothèses de 2) complétée par une production sous forme thermique suivant celles de 1).

Les résultats obtenus apparaissent dans le tableau VI.

\section{Tableau VI}

$\begin{array}{clllll}n & 16 & 100 & 1.600 & 10000 & 90000 \\ \overline{1)} \tau & \overline{3,33} & \overline{3,17} & \overline{2,96} & \overline{2,78} & <-2,5\left(^{2}\right) \\ \eta_{\mathrm{e}} & 0,1 & 0,2 & 0,3 & 0,35 & 0,4 \\ 2) \tau & 3,19 & 3,17 & 3,06 & 2,94 & 2,71 \\ 3) \tau & 3,38 & 3,25 & 3,11 & 2,99 & 2,76\end{array}$

$\left({ }^{2}\right)$ La valeur $\tau=2,5$ est considérée comme celle conduisant au rapprochement maximal des capteurs.

Pour les cas 2) et 3), il a été procédé ainsi : du graphe de la figure 11 , on déduit la valeur réelle de $C_{n}$ d'après (Fig. 11) :

$$
\begin{aligned}
C_{n}^{*}= & A_{1} A_{4}+\frac{A_{4} A_{5}}{5 \eta_{\mathrm{e}}}+\left(A_{5} A_{6}+\frac{A_{6} A_{7}}{5 \eta_{\mathrm{e}}}\right) \times \\
& \times \frac{1}{F S_{\alpha, \beta} \eta_{\mathrm{e}}}
\end{aligned}
$$

et l'on reporte cette valeur sur le graphe de la figure 10 en l'égalant à celle de $(\delta \xi / \delta \tau)^{*}$. Il en découle la valeur du coefficient d'espacement optimisé. Si l'on avait envisagé un coût du terrain $\left(F_{\mathrm{s}}\right)$ différent de $10 \mathrm{~F}$, il aurait précédemment fallu déterminer les variations réelles de $(\delta \xi / \delta \tau)^{*}$ comme suit (voir Fig. 10) :

$$
\left(\frac{\delta \xi}{\delta \tau}\right)^{*}=B_{1} B_{3}-B_{2} B_{3} \frac{F_{\mathrm{s}}}{10}
$$

4. Conclusion. - A condition qu'elle soit implantée sur un site météorologiquement comparable à celui de Carpentras, il nous est maintenant possible d'optimiser le réseau de transfert thermique, défini paragraphe 2, d'une centrale solaire de type THEK, quels que soient sa nature et son dimensionnement De plus, au cours de cette étude, ont été employées des démarches de calcul utilisables quels que soient le site d'implantation et la nature des héliostats. Le mode de connexion proposé et les moyens de détermination de ses caractéristiques, ainsi que la présentation du coût et des pertes énergétiques du réseau de transfert sous forme linéaire nous semblent, en particulier, être d'une généralité satisfaisante. La méthode d'optimisation employée présente en outre l'avantage de ne faire appel qu'à un minimum de données météorologiques (paragraphes 1 et 3 ) et de ne nécessiter un support informatique important, au moins pour les grandes centrales, que dans l'étude $\mathrm{du}$ réseau de transfert lui-même (paragraphe 2). Or, ce dernier ne dépend que faiblement du site choisi. Ce qui constitue donc l'aspect le plus limitatif du travail présenté (le choix du site) ne semble pas être un obstacle à son exploitation, ou à celle d'une étude comparable qui concernerait des héliostats différents.

On peut, en dernier lieu, se poser la question de savoir quelles sont les performances des centrales solaires dont nous avons optimisé le réseau de transfert thermique. Dans le tableau 7 sont données quelques caractéristiques des divers types de centrales dont les coefficients d'espacement optimisés apparaissent dans le tableau 6.

Remarquons que notre objet n'était pas ici de prendre en considération les facteurs limitatifs propres aux centrales solaires de toutes natures; or, ceux-ci pourraient s'avérer les plus contraignants : on doit en particulier penser aux problèmes soulevés par un éventuel stockage de l'énergie, ou par la bonne gestion du champ.

Si l'on fait abstraction de cette remarque, on peut essayer d'évaluer, d'après les résultats que nous avons obtenus, le dimensionnement optimal des centrales solaires étudiées ici.

Dans l'hypothèse d'une production d'énergie sous forme thermique, les unités les plus importantes ne semblent pas présenter un grand intérêt. Il ne faut alors toutefois pas sous-estimer l'argument économique : la centrale doit comporter un nombre d'héliostats tel qu'une fabrication industrielle, au moins en petite série, puisse être envisagée. 


\begin{tabular}{|c|c|c|c|c|c|c|c|c|c|c|}
\hline \multirow[b]{3}{*}{$\begin{array}{c}\text { Type } \\
\text { de centrale }\end{array}$} & \multicolumn{6}{|c|}{ Pertes énergétiques réseau $(\%)$} & \multicolumn{4}{|c|}{ Caractéristiques centrales $\left({ }^{3}\right)$} \\
\hline & \multicolumn{6}{|c|}{ Inertie } & \multirow{2}{*}{$\begin{array}{c}\text { Bilan (\%) } \\
\text { thermique } \\
\text { annuel }\end{array}$} & \multirow{2}{*}{$\begin{array}{c}\text { Bilan (\%) } \\
\text { électrique } \\
\text { annuel }\end{array}$} & \multirow{2}{*}{$\begin{array}{l}\text { Production } \\
\text { thermique/an } \\
\text { (MWh) }\end{array}$} & \multirow{2}{*}{$\begin{array}{c}\text { Production } \\
\text { électrique/an } \\
\text { (MWh) }\end{array}$} \\
\hline & $\begin{array}{c}\text { Effets } \\
\text { d'ombre }\end{array}$ & Ligne & $\begin{array}{l}\text { nuits } \\
+\mathrm{m} \cdot \mathrm{j}\end{array}$ & $\begin{array}{l}\text { nua- } \\
\text { ges }\end{array}$ & $\begin{array}{l}\text { Pompage } \\
\text { pondéré }\end{array}$ & $\sum$ réseau & & & & \\
\hline- & - & - & - & - & - & - & - & - & - & - \\
\hline \multicolumn{11}{|l|}{ 1) } \\
\hline 16 & 6,45 & 8,85 & 1,70 & 1,45 & 0,78 & 19,23 & 51,63 & & 687 & \\
\hline 100 & 7,60 & 10,14 & 2,12 & 1,68 & 1,03 & 22,57 & 48,69 & & $4,05 \times 10^{3}$ & \\
\hline 1600 & 9,60 & 11,07 & 2,85 & 1,97 & 2,11 & 27,60 & 44,35 & & $59,0 \times 10^{3}$ & \\
\hline 10000 & 11,80 & 11,42 & 3,46 & 2,18 & 4,23 & 33,09 & 39,62 & & $329 \times 10^{3}$ & \\
\hline 90000 & 16,50 & 11,81 & 4,66 & 2,52 & 10,23 & 45,72 & 28,63 & & $2140 \times 10^{3}$ & \\
\hline \multicolumn{11}{|c|}{ 2) } \\
\hline 16 & 7,40 & 8,66 & 1,67 & 1,43 & 1,54 & 20,70 & & 5,05 & & 67,1 \\
\hline 100 & 7,60 & 10,14 & 2,12 & 1,68 & 1,03 & 22,57 & & 9,74 & & 810 \\
\hline 1600 & 8,60 & 11,30 & 2,92 & 2,01 & 1,44 & 26,27 & & 13,61 & & $18,1 \times 10^{3}$ \\
\hline 10000 & 9,80 & 11,82 & 3,61 & 2,26 & 2,54 & 30,03 & & 14,71 & & $122 \times 10^{3}$ \\
\hline 90000 & 12,80 & 12,43 & 4,98 & 2,66 & 5,52 & 38,39 & & 13,89 & & $1040 \times 10^{3}$ \\
\hline \multicolumn{11}{|c|}{ 3) } \\
\hline 16 & 6,15 & 8,92 & 1,71 & 1,46 & 1,56 & 19,80 & 45,88 & 5,10 & 610 & 67,8 \\
\hline 100 & 7,00 & 10,28 & 2,15 & 1,70 & 1,04 & 22,17 & 39,13 & 9,78 & $3,25 \times 10^{3}$ & 813 \\
\hline 1600 & 8,10 & 11,41 & 2,95 & 2,03 & 1,46 & 25,95 & 31,87 & 13,66 & $42,4 \times 10^{3}$ & $18,2 \times 10^{3}$ \\
\hline 10000 & 9,30 & 11,95 & 3,65 & 2,28 & 2,57 & 29,75 & 27,40 & 14,76 & $228 \times 10^{3}$ & $123 \times 10^{3}$ \\
\hline 90000 & 12,05 & 12,58 & 5,06 & 2,69 & 5,61 & 37,99 & 20,94 & 13,96 & $1570 \times 10^{3}$ & $1040 \times 10^{3}$ \\
\hline
\end{tabular}

$\left(^{3}\right)$ On a considéré, dans la détermination de ces caractéristiques, les centrales comme non équipées d'un réservoir de stockage.

Dans l'hypothèse d'une production d'énergie totale ou électrique, c'est la prise en considération des convertisseurs thermo-électriques existants sur le marché qui contribuera à la meilleure évaluation du dimensionnement optimal. L'obtention de rendements relativement élevés n'est actuellement envisageable que pour des centrales comportant un nombre important de capteurs; on est alors conduit à des puissances électriques crête (pour une puissance solaire incidente de $800 \mathrm{~W} / \mathrm{m}^{2}$ ) de l'ordre de quelques centaines de $\mathrm{kWe}$ à quelques dizaines de MWe.

A ce jour, les centrales à convertisseurs héliothermiques distribués, en particulier celles étudiées ici, semblent pouvoir couvrir une large gamme de puissance dont la valeur crête maximale serait très approximativement de l'ordre de $200 \mathrm{MWt}$. Cependant, même dans l'hypothèse d'une production d'énergie sous forme électrique ou totale, les unités de moyenne puissance seront, à l'avenir, vraisemblablement, les plus performantes.

Annexe I. - La valeur du coefficient d'isolation $(A)$ est déterminée en minimisant la somme des termes :

- investissement correspondant à l'isolation,

- valeur monétaire actualisée correspondant aux pertes thermiques en ligne.

Dans le cas d'une production de chaleur, avec les notations du paragraphe 3 et du fait des hypothèses émises, cette somme $\left(\sum\right)$, par unité de longueur, s'écrit : $\sum=v_{\mathrm{i}} F_{\mathrm{i}}+p_{l} t_{\mathrm{f}} F S_{\alpha, \beta}$.

Sa minimisation conduit à l'expression de $A$ :

$A=-\frac{F_{\mathrm{i}}}{t_{\mathrm{f}} F S_{\alpha, \beta}}$.
Pratiquement, par souci de simplification, nous avons utilisé dans notre étude une valeur de $A$ constante. Ce paramètre sert alors à l'homogénéisation des épaisseurs d'isolant utilisées.

Annexe II. - L'expression de $W_{\mathrm{i}}$ s'établit comme suit :

1) Pour le terme qui correspond aux nuits et aux séquences de mauvais jours : on a :

$\sum_{j=1}^{j=365} j N_{j}=N$

Or, pour des valeurs de $j$ encore très inférieures à 365 , la valeur de $N_{j}$ est approximativement nulle. Cette égalité peut donc s'écrire :

$\sum_{j=1}^{j=\infty} j N_{j}=N$

par ailleurs, si $p \neq 1$ :

$N_{j}=p N_{j-1}=N_{1} p^{j-1}$

il vient donc :

$N_{1} \sum_{j=1}^{j=\infty} j p^{j-1}=N$

or :

$\sum_{j=1}^{j=\infty} j p^{j-1}=\frac{1}{(1-p)^{2}}$

d'où :

$N_{1}=(1-p)^{2} N$ 
et

$N_{j}=(1-p)^{2} N p^{j-1}=365(1-p)^{2} p^{j}$.

A une séquence de $j$ mauvais jours correspond l'effet de l'inertie thermique associée à une interruption de fonctionnement de la centrale approximativement égale à $(2 j+1) 12$ heures, si l'on raisonne sur une journée de type équinoxe et si l'on néglige le temps de non-fonctionnement induit par la condition $H<H_{\mathrm{s}}$. D'où l'expression de l'inertie thermique due aux séquences de mauvais jours :

$\sum_{j=1}^{j=\infty} N_{j} W_{\mathrm{t}=(2 j+1) 12 \text { heures }}$

en remarquant que pour $j=0$, cette expression devient $N_{0} W_{\mathrm{t}=12 \text { heures }}$ on pose, $N_{0}$ égal au nombre de nuits de l'année auquel on a retranché le nombre de nuits déjà incluses dans les séquences de mauvais jours ; ainsi apparaît le premier terme de (21). Du fait de la définition de $N_{0}$, on peut écrire :

$N_{0}=365-\sum_{j=1}^{j=\infty}(j+1) N_{j}$

soit :

$N_{0}=365-N(2-p)$.

2) Pour le terme qui correspond aux passages nuageux : la durée maximale de ce passage nuageux est ici inférieure à $(12-m)$ heures, car, dans le cas contraire, il n'y a pas fonctionnement de la centrale et cette hypothèse vient d'être envisagée.

Supposons que la durée d'un nuage soit discrétisable et donc égale à un multiple $k \Delta t$ d'un temps d'interruption $\Delta t$; le nombre de durées possibles $(K)$, $m$ et $\Delta t$ étant exprimés en heures, est égal à :

$K=\frac{(12-m)}{\Delta t}$

$\mathrm{Vu}$ les hypothèses énoncées au paragraphe 3 , au nuage de durée $k \Delta t$ est associé un temps de noninsolation égal à : $t_{n} / K$ et le nombre de fois où ce nuage apparaîtra est égal à $t_{n} / K k \Delta t$.

L'expression correspond à l'inertie thermique due aux passages nuageux pendant le fonctionnement de la centrale s'écrit :

$$
\begin{aligned}
& \sum_{k=1}^{k=K} \frac{t_{n}}{K k \Delta t} W_{\mathrm{t}=k \Delta t}= \\
& \quad=\sum_{k=1}^{k=K} \frac{t_{n} \Delta t}{(12-m) k \Delta t} W_{\mathrm{t}=k \Delta t}
\end{aligned}
$$

En faisant tendre $\Delta t$ vers 0 , on trouve le second membre de (20) :

$\frac{t_{n}}{12-m} \int_{0}^{12-m} \frac{W_{t}}{t} \mathrm{~d} t$.

Le nombre de nuages possibles est infini et, d'après les hypothèses émises, aucun de ces nuages n'est prépondérant.

\section{Bibliographie}

[1] Programme THEK, C.N.R.S.-PIRDES, Dpt d'Héliophysique, Université de Provence, Marseille, septembre 1977.

[2] Perrin de Brichambaut, Ch., Estimation des ressources énergétiques solaires, Journées d'Etude de l'ESIM, 2627 juin 1974

[3] Bedeil, J., Ceron, J. P., JAN, J., Le rayonnement solaire à
Carpentras, Secrétariat d'Etat auprès du Ministre de l'Equipement et de l'Aménagement du Territoire, Division Climatologie, octobre 1977.

[4] LestienNe, R., Modèle Markovien simplifié de météorologie à deux états, l'exemple d'Odeillo, la Météorologie (1978), pp. 53-63. 